Proyecciones Journal of Mathematics

Vol. 38, No 2, pp. 305-314, June 2019.

Universidad Católica del Norte

Antofagasta - Chile

\title{
Graceful centers of graceful graphs and universal graceful graphs
}

\author{
H. M. Makadia \\ Lukhdhirji Engineering College, India \\ H. M. Karavadiya \\ Labhubhai Trivedi Institute of Technology, India \\ and \\ V. J. Kaneria \\ Saurashtra University, India \\ Received: September 201\%. Accepted : December 2018
}

\begin{abstract}
In this paper we define graceful center of a graceful graph. We proved any graph $G$ which admits $\alpha$-labeling has at least four graceful centers. We also defined a new strong concept of universal graceful graph. Some results on ring sum of two graphs for their graceful labeling are proved.
\end{abstract}

Key words: Graceful center of a graceful graph, universal graceful labeling, ring sum of two graphs.

AMS Subject Classification Number: $05 C^{\prime 2} 8$. 


\section{Introduction}

In this paper a $(p, q)$ graph $G$, we mean $|V(G)|=p,|E(G)|=q$ and it is a finite, undirected simple graph. Terms not defined here are used from Harary [3]. Rosa [1] introduced the notion of graceful labeling ( $\beta$ valuation) and $\alpha$-labeling of a graph. Any graph $G$, which admits $\alpha$-labeling is necessarily a bipartite graph; such graph is known as $\alpha$-graceful graph.

A Graph $G=(V, E)$ is said to be a graceful graph if $G$ admits a function $f: V(G) \longrightarrow\{0,1, \ldots, q\}$ is injective and the induce edge function $f^{\star}: E(G) \longrightarrow\{1,2, \ldots, q\}$ denoted by $f^{\star}(e=u v)=|f(u)-f(v)|$ is bijective, $\forall e=u v \in E$. Here function $f$ is called graceful labeling of graph $G$.

Let $G$ be a graceful graph with a graceful labeling $f: V(G) \longrightarrow$ $\{0,1,2, \ldots, q\}$. A vertex $v \in V(G)$ is called a graceful center of $G$ if $f(v)=0$ or $f(v)=q$. Any graceful graph $G$ with graceful labeling $f$ has at least two graceful centers. It is obvious that $q-f$ is also a graceful labeling for $G$ and it produce same graceful centers for $G$. If a graph $G$ has precisely two graceful centers, then they are adjacent in $G$, as they produce the edge label $q$ under $f$.

A graph $G$ is said to be a universal graceful graph if for any $v \in V(G)$, $v$ is a graceful center for $G$ with respect to some graceful labeling of $G$. Also we call $G$ is a universal $\alpha$-graceful graph if for any $v \in V(G), v$ is a graceful center for $G$ with respect to some $\alpha$-graceful labeling of $G$.

Every cycle $C_{n}(n \equiv 0(\bmod 4))$, star $K_{1, n}$ are universal graceful graphs as well they are universal $\alpha$-graceful graphs. While $C_{n}(n \equiv 3(\bmod 4))$ and wheel $W_{n}$ are universal graceful graphs, but they are not universal $\alpha$-graceful graphs, as symmetric structure of above said graphs and their graceful labeling are given in Rosa [1], Hoede and Kuiper [2].

Ring sum of two graphs $G_{1}$ and $G_{2}$ denoted $G_{1} \oplus G_{2}$, where $G_{1} \oplus G_{2}=$ $\left(V\left(G_{1}\right) \cup V\left(G_{2}\right), E\left(G_{1}\right) \cup E\left(G_{2}\right)-E\left(G_{1}\right) \cap E\left(G_{2}\right)\right)$. Throughout this paper we consider the ring sum of a graceful graph $G$ with $K_{1, n}$ by considering one vertex $v$ which is a graceful center of $G$ and the apex vertex of $K_{1, n}$ as a common vertex. Rest vertex of $G$ and $K_{1, n}$ are distinct. If $H=G \oplus K_{1, n}$ then $H=\left(V(G) \cup V\left(K_{1, n}\right), E(G) \cup E\left(K_{1, n}\right)\right)$, as $E(G) \cap E\left(K_{1, n}\right)=\phi$. Thus, $|V(H)|=|V(G)|+n$ and $|E(H)|=|E(G)|+n$. 


\section{Main Results}

Theorem - 1 : Any $\alpha$-graceful graph $G$ has atleast four graceful centers.

Proof : Let $G$ be an $\alpha$-graceful graph and $f: V(G) \longrightarrow\{0,1,2, \ldots, q\}$ be an $\alpha$-labeling for $G$. Since, $f$ is $\alpha$-labeling for $G$ then $\exists$ an integer $k(0 \leq k<q)$ such that for any $u v \in E(G), \min \{f(u), f(v)\} \leq k<$ $\max \{f(u), f(v)\}$. Thus, $V(G)$ can be partitioned into two parts

$\mathrm{V}_{1}=\{v \in V(G) / f(v) \leq k\}$ and

$V_{2}=V(G)-V_{1}=\{v \in V(G) / f(v)>k\}$.

Take $\left|V_{1}\right|=l$. It is obvious that $\left|V_{2}\right|=p-l$. Moreover, $\exists w_{1}, w_{2} \in V_{1}$ such that $f\left(w_{1}\right)=0, f\left(w_{2}\right)=k$ and $\exists w_{3}, w_{4} \in V_{2}$ such that $f\left(w_{3}\right)=k+1$ and $f\left(w_{4}\right)=q$.

Here $w_{1}$ and $w_{4}$ both are graceful centers for $G$ with respect to $\alpha$ graceful labeling $f$.

Define $h: V(G) \longrightarrow\{0,1,2, \ldots, q\}$ as follows.

$\mathrm{h}(\mathrm{u})=\mathrm{k}-\mathrm{f}(\mathrm{u}), \forall v \in V_{1}$ and

$h(v)=q+k+1-f(v), \forall v \in V_{2}$.

Note that $h$ is injective, as $f$ is an injective map. Further for any $u v \in E(G)$

$$
\mathrm{h}^{\star}(u v)=|h(u)-h(v)|
$$

$=h(v)-h(u)$, assuming $u \in V_{1}$

$=q+k+1-f(v)-k+f(u)$

$=q+1-(f(v)-f(u))$

$=q+1-f^{\star}(u v)$.

Therefore, $h^{\star}: E(G) \longrightarrow\{1,2, \ldots, q\}$ is also a bijection, as $f^{\star}$ is a bijective map. Thus, $h$ is also a graceful labeling for $G$. Infact $h$ is an $\alpha$-graceful labeling for $G$, as $\min \{h(u), h(v)\} \leq k \leq \max \{h(u), h(v)\}, \forall$ $u v \in E(G)$. Since, $h\left(w_{2}\right)=0$ and $h\left(w_{3}\right)=q, w_{2}$ and $w_{4}$ are graceful centers for $G$ with respect to $\alpha$-labeling $h$. Thus, $G$ has graceful centers $w_{1}, w_{2}, w_{3}$ and $w_{4}$. So, $G$ admits atlest four graceful centers.

Remark : A $\alpha$-graceful graph $G$ with $\alpha$-labeling $f$ admits three more $\alpha$-labelings $q-f, h$ and $q-h$, as discussed in last theorem.

Theorem-2 : If $G$ is a graceful graph, then $G \oplus K_{1, n}$ is also a graceful graph, for all $n \in N$. 
Proof : Let $f: V(G) \longrightarrow\{0,1,2, \ldots, q\}$ be a graceful labeling for $G$ and $v \in V(G)$ such that $f(v)=0$. i.e. $v$ is a graceful center for $G$ with respect to $f$.

Let $H=G \oplus K_{1}$ by considering vertex $v$ of $G$ and the apex vertex of $K_{1, n}$ as a common vertex in $H$. Let $V(G)=\left\{v_{1}, v_{2}, \ldots, v_{p}=v\right\}$ and $V\left(K_{1, n}\right)=\left\{v, u_{1}, u_{2}, \ldots, u_{n}\right\}$ with $v$ is the apex vertex of $K_{1, n}$. It is obvious that $V(H)=V(G) \cup\left\{u_{1}, u_{2}, \ldots, u_{n}\right\}$ and $E(H)=E(G) \cup\left\{v u_{i} / 1 \leq i \leq n\right\}$. i.e. $|V(H)|=p+n$ and $|E(H)|=q+n$.

Without loss of generality we assume here $f(v)=0$. Otherwise $f(v)=q$ and in this case $q-f$ is a graceful labeling for $G$ with $(q-f)(v)=0$. In this case $v$ is also a graceful center for $G$ with respect to $q-f$.

Define $h: V(H) \longrightarrow\{0,1,2, \ldots, q+n\}$ as follows.

$\mathrm{h}(\mathrm{w})=\mathrm{f}(\mathrm{w}), \forall w \in V(G)$ and $h\left(u_{i}\right)=q+i, \forall i=1,2, \ldots, n$.

Note that $h$ is an injective map, as $f$ is injective. Also for any $u w \in$ $E(H), h^{\star}(u w)=f^{\star}(u w) \in\{1,2, \ldots, q\}$, if $u w \in E(G)$ and $h^{\star}(u w)=$ $h^{\star}\left(v u_{i}\right)=q+i, \forall i=1,2, \ldots, n$, if $u w \in E\left(K_{1, n}\right)$ (assuming $u=v$ and $w=u_{i}$, for some $\left.i \in\{1,2, \ldots, n\}\right)$. Therefore range of $h^{\star}$ is $\{1,2, \ldots, q+n\}$ and so, it is a bijective map. Hence, $h$ is a graceful labeling for $H$ and $H$ is a graceful graph, for all $n \in N$.

Corollary - 2.1 : $\quad C_{n} \oplus K_{1, t}$ is graceful, where $t \in N$ and $n \equiv 0,3(\bmod$ $4)$.

Corollary - 2.2 : $\quad W_{n} \oplus K_{1, t}$ is graceful, $\forall t, n \in N$.

Theorem - 3 : If $G$ is a universal graceful graph, then its one vertex super graph $G \oplus K_{2}$ is a graceful graph.

Proof : Let $v \in V(G)$ be any fixed vertex. Since, $G$ is a universal graceful graph, there is a graceful labeling $f: V(G) \longrightarrow\{0,1,2, \ldots, q\}$ such that $f(v)=0$.

Let $H=G \oplus K_{2}$, the ring sum of $G$ with $K_{2}$ by considering vertex $v$ and one pendant vertex of $K_{2}$ as a common vertex.

It is obvious that $|V(H)|=|V(G)|+1$ and $|E(H)|=|E(G)|+1$. Let $V(H)=V(G) \cup\{w\}$. Then we see that $E(H)=E(G) \cup\{v w\}$, as $v$ and $w$ are adjacent vertices of $K_{2}$.

Define $h: V(H) \longrightarrow\{0,1, \ldots,|E(H)|\}$ as follows. 
$\mathrm{h}(\mathrm{w})=\mathrm{q}+1$ and

$\mathrm{h}(\mathrm{u})=\mathrm{f}(\mathrm{u}), \forall u \in V(G)$, where $q=|E(G)|$.

It is observed that $h$ is an injective map as $f$ is injective. Moreover $\mathrm{h}^{\star}(u w)=h(w)-h(v)$

$=q+1-f(v)$

$=q+1-0$

$=q+1$ and for any $u_{1} u_{2} \in V(G)$

$h^{\star}\left(u_{1} u_{2}\right)=\left|h\left(u_{1}\right)-h\left(u_{2}\right)\right|$

$=\left|f\left(u_{1}\right)-f\left(u_{2}\right)\right|$

$=f^{\star}\left(u_{1} u_{2}\right)$.

Therefore, $h^{\star}: E(H) \longrightarrow\{1,2, \ldots,|E(H)|\}$ is bijective and so, $h$ becomes a graceful labeling for $H$. Thus, $G \oplus K_{2}$ is a graceful graph.

Theorem - 4 : Let $G_{1}$ be a graceful graph and $G_{2}$ be an $\alpha$-graceful graph. Then ring sum $G_{1} \oplus G_{2}$ by considering graceful center of $G_{1}$ and the graceful center of $G_{2}$ as a common vertex is a graceful graph.

Proof : Let $f_{1}: V\left(G_{1}\right) \longrightarrow\left\{0,1, \ldots, q_{1}\right\}$ be a graceful labeling and $f_{1}\left(w_{1}\right)=0$, for some $w_{1} \in V\left(G_{1}\right)$, where $q_{1}=\left|E\left(G_{1}\right)\right|$. Since, $G_{2}$ is an $\alpha$-graceful graph, $\exists f_{2}: V\left(G_{2}\right) \longrightarrow\left\{0,1, \ldots, q_{2}\right\}$ a graceful labeling for $G_{2}$ and an integer $k\left(0 \leq k<q_{2}\right)$ such that for each $u v \in E\left(G_{2}\right)$, $\min \left\{f_{2}(u), f_{2}(v)\right\} \leq k<\max \left\{f_{2}(u), f_{2}(v)\right\}$, where $q_{2}=\left|E\left(G_{2}\right)\right|$. Let $f_{2}\left(w_{2}\right)=0$, where $w_{2} \in V\left(G_{2}\right)$. Take $H=G_{1} \oplus G_{2}$ by considering $w_{1}$ and $w_{2}$ as a common vertex. It is obvious that $E(H)=E\left(G_{1}\right) \cup E\left(G_{2}\right)$, $|E(G)|=q_{1}+q_{2}$.

Define $g: V(H) \longrightarrow\left\{0,1, \ldots, q_{1}+q_{2}\right\}$ as follows:

$\mathrm{g}=\mathrm{k}-\mathrm{f}_{2}$ on $V_{1}$,

$g=q_{1}+q_{2}+k+1-f_{2}$ on $V_{2}$ and

$g=k+f_{1}$ on $V\left(G_{1}\right)$, where

$V_{1}=\left\{w \in V\left(G_{2}\right) / f_{2}(w) \leq k\right\}$ and $V_{2}=V\left(G_{2}\right)-V_{1}$.

Since, range of $g$ on $V_{1} \subseteq\{0,1,2, \ldots, k\}$, range of $g$ on $V_{2} \subseteq\left\{q_{1}+k+\right.$ $\left.1, q_{1}+k+2, \ldots, q_{1}+q_{2}\right\}$ and range of $g$ on $V(G) \subseteq\left\{k+1, k+2, \ldots, k+q_{1}\right\}$, $g$ is a one-one map.

Moreover

$$
g^{\star}=f_{1}^{\star} \text { on } E\left(G_{1}\right) \text { and } g^{\star}=q_{1}+f_{2}^{\star} \text { on } E\left(G_{2}\right) .
$$

Thus, range of $g^{\star}=\left\{1,2, \ldots, q_{1}, q_{1}+1, \ldots, q_{1}+q_{2}\right\}$ and so, it is a bijective map. Therefore, $g$ is a a graceful labeling for $H$ and so, $H=G \oplus G_{2}$ is a graceful graph. 
Theorem - 5 : If $G_{1}$ and $G_{2}$ be two $\alpha$-graceful graphs, then the ring sum $G_{1} \oplus G_{2}$ considering two graceful centers of $G_{1}$ and $G_{2}$ as a common vertex is an $\alpha$-graceful graph.

Proof : $\quad$ Since, $G_{1}$ and $G_{2}$ both are $\alpha$-graceful graphs, $\exists f_{i}: V\left(G_{i}\right) \longrightarrow$ $\left\{0,1,2, \ldots, q_{i}\right\}$ graceful labeling for $G_{i}$ and non-negative integer $k_{i}(0 \leq$ $\left.k_{i}<q_{i}\right)$ such that for each $u v \in E\left(G_{i}\right), \min \left\{f_{i}(u), f_{i}(v)\right\} \leq k_{i}<\max \left\{f_{i}(u), f_{i}(v)\right\}$, where $q_{i}=\left|E\left(G_{i}\right)\right|$ and $i=1,2$.

Let $f_{1}\left(w_{1}\right)=0, f_{2}\left(w_{2}\right)=0$, where $w_{i} \in V\left(G_{i}\right), i=1,2$. Take $H=$ $G_{1} \oplus G_{2}$ by considering $w_{1}$ and $w_{2}$ as a common vertex. It is obvious that $E(H)=E\left(G_{1}\right) \cup E\left(G_{2}\right)$ and $|E(H)|=q_{1}+q_{2}$.

Take $V_{1}=\left\{w \in V\left(G_{1}\right) / f_{1}(w) \leq k_{1}\right\}, V_{2}=V\left(G_{1}\right)-V_{1}, V_{3}=\{w \in$ $\left.V\left(G_{2}\right) / f_{2}(w) \leq k_{2}\right\}$ and $V_{4}=V\left(G_{2}\right)-V_{3}$.

Define $g: V(H) \longrightarrow\left\{0,1,2, \ldots, q_{1}+q_{2}\right\}$ as follows.

$\mathrm{g}=\mathrm{k}_{2}-f_{2}$ on $V_{3}$,

$=q_{1}+q_{2}+k_{2}+1-f_{2}$ on $V_{4}$ and

$=k_{2}+f_{1}$ on $V\left(G_{1}\right)$.

Since, range of $g$ on $V_{3} \subseteq\left\{0,1,2, \ldots, k_{2}\right\}$ range of $g$ on $V_{4} \subseteq\left\{q_{1}+k_{2}+\right.$ $\left.1, q_{1}+k_{2}+2, \ldots, q_{1}+q_{2}\right\}$ and range of $g$ on $V\left(G_{1}\right) \subseteq\left\{k_{2}+1, k_{2}+2, \ldots, k_{2}+\right.$ $\left.q_{1}\right\}, g$ is a one-one map. Moreover, $g^{\star}=f_{1}^{\star}$ on $E\left(G_{1}\right)$ and $g^{\star}=g_{1}+f_{2}^{\star}$ on $E\left(G_{2}\right)$ gives range of $g^{\star}=\left\{1,2, \ldots, q_{1}, q_{2}+1, \ldots, q_{1}+q_{2}\right\}$. Therefore, $g^{\star}$ is a bijective map and so, it is a graceful labeling for $H=G_{1} \oplus G_{2}$.

Take $k=k_{1}+k_{2}$. Let $u v \in E(H)$ be any edge.

$\Rightarrow$ Either $u v \in E\left(G_{1}\right)$ or $u v \in E\left(G_{2}\right)$.

Case-I : $ı u v \in E\left(G_{1}\right)$.

Without loss of generality we assume here $u \in V_{1}$ and $v \in V_{2}$. Now $g(u)=k_{2}+f_{1}(u) \leq k_{2}+k_{1}=k$ and $g(v)=k_{2}+f_{1}(v)>k_{2}+k_{1}=k$.

Case-II : $1 u v \in E\left(G_{2}\right)$.

Without loss of generality we assume here $u \in V_{3}$ and $v \in V_{4}$. Now $g(u)=k_{2}-f_{2}(u) \leq k_{2}$ and $g(v)=q_{1}+q_{2}+k_{2}+1-f_{2}(v)=q_{1}+k_{2}+1+$ $\left(q_{2}-f_{2}(v)\right)<k$, as $q_{2}-f_{2}(v) \geq 0$ and $k_{1}<q_{1}$.

Thus, for any case we get $\min \{g(u), g(v)\} \leq k<\max \{g(u), g(v)\}, \forall$ $u v \in E(H)$.

Hence, $h$ is an $\alpha$-graceful labeling for $H$ and so, $H=G_{1} \oplus G_{2}$ is an $\alpha$-graceful graph.

Here four graceful centers of $G_{1} \oplus G_{2}$ are $w_{3}, w_{4}, w_{5}, w_{6}$, where $f_{1}\left(w_{3}\right)=$ $k_{1}, f_{1}\left(w_{4}\right)=k_{1}+1, f_{2}\left(w_{5}\right)=k_{2}$ and $f_{2}\left(w_{6}\right)=k_{2}+1, w_{3}, w_{4} \in V\left(G_{1}\right)$, $w_{5}, w_{6} \in V\left(G_{2}\right)$. Because

$$
\mathrm{g}\left(\mathrm{w}_{3}\right)=k_{2}+f_{1}\left(w_{3}\right)=k_{2}+k_{1}=k,
$$


$g\left(w_{4}\right)=k_{2}+f_{1}\left(w_{4}\right)=k_{2}+k_{1}+1=k+1$,

$g\left(w_{5}\right)=k_{2}-f_{2}\left(w_{5}\right)=k_{2}-k_{2}=0$ and

$g\left(w_{6}\right)=q_{1}+q_{2}+k_{2}+1-f_{2}\left(w_{6}\right)=q_{1}+q_{2}$.

Now we give a counter example which is $\alpha$-graceful but not universal graceful graph; Namely a special type of caterpillar.

A caterpillar is a tree with the property that the removal of its pendant vertices leaves a path. This path is known as spine of the caterpillar. It is denoted by $S\left(n_{1}, n_{2}, \ldots, n_{k}\right)$, where $P_{k}$ is the spine of the given caterpillar and $n_{1}, n_{2}, \ldots, n_{k}$ are number of pendant vertices, which are adjacent with the spine of $S\left(n_{1}, n_{2}, \ldots, n_{k}\right)$.

Theorem - 6 : $\quad$ Let $T$ be a caterpillar $S(2,0,1)$. Then $T$ be an $\alpha$-graceful graph, but it is not a universal graceful graph, as the vertex $v$ can not be a graceful center for $T$ with respect to any graceful labeling for $T$.

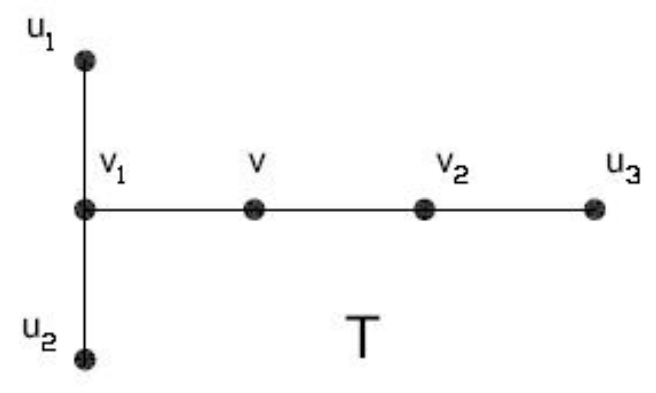

Proof : As above tree $T$ is a caterpillar $S(2,0,1)$, it is an $\alpha$-graceful graph.

Suppose $T$ admits a graceful center $v$ with respect to a graceful labeling $f$ on $T$ if possible. Here $f(v)=0$ and $v$ is adjacent to one vertex whose vertex label is $q=5$. i.e. there are two cases either $f\left(v_{1}\right)=5$ or $f\left(v_{2}\right)=5$. In there both cases remaining four vertices have following $24-24$ possibilities are given in following table-1 and table-2.

From these table $f$ creates one edge label twice and so, in any case $f$ can not be a graceful labeling for $T$.

Therefore, $T$ is not a universal graceful tree. 


\begin{tabular}{||c|c|c|c|c||}
\hline \hline$f\left(v_{2}\right)$ & $f\left(u_{1}\right)$ & $f\left(u_{2}\right)$ & $f\left(u_{3}\right)$ & Possible four edge labels \\
\hline \hline 1 & 2 & 3 & 4 & $1,3,3,2$ \\
\hline 1 & 2 & 4 & 3 & $1,2,3,1$ \\
\hline 1 & 3 & 2 & 4 & $1,3,3,2$ \\
\hline 1 & 3 & 4 & 2 & $1,1,2,1$ \\
\hline 1 & 4 & 2 & 3 & $1,2,1,3$ \\
\hline 1 & 4 & 3 & 2 & $1,1,1,2$ \\
\hline 2 & 1 & 3 & 4 & $2,2,4,2$ \\
\hline 2 & 1 & 4 & 3 & $2,1,4,1$ \\
\hline 2 & 3 & 1 & 4 & $2,2,2,4$ \\
\hline 2 & 3 & 4 & 1 & $2,1,2,1$ \\
\hline 2 & 4 & 1 & 3 & $2,1,1,4$ \\
\hline 2 & 4 & 3 & 1 & $2,1,1,2$ \\
\hline 3 & 1 & 2 & 4 & $3,1,4,3$ \\
\hline 3 & 1 & 4 & 2 & $3,1,4,1$ \\
\hline 3 & 2 & 1 & 4 & $3,1,3,4$ \\
\hline 3 & 2 & 4 & 1 & $3,2,3,1$ \\
\hline 3 & 4 & 1 & 2 & $3,1,1,4$ \\
\hline 3 & 4 & 2 & 1 & $3,2,1,3$ \\
\hline 4 & 1 & 2 & 3 & $4,1,4,3$ \\
\hline 4 & 1 & 3 & 2 & $4,2,4,2$ \\
\hline 4 & 2 & 1 & 3 & $4,1,3,4$ \\
\hline 4 & 2 & 3 & 1 & $4,3,3,2$ \\
\hline 4 & 3 & 1 & 2 & $4,2,2,4$ \\
\hline 4 & 3 & 2 & 1 & $4,3,2,3$ \\
\hline \hline & & & & \\
\hline
\end{tabular}

Table-1: If $f\left(v_{1}\right)=5$ 


\begin{tabular}{||c|c|c|c|c||}
\hline \hline$f\left(v_{2}\right)$ & $f\left(u_{1}\right)$ & $f\left(u_{2}\right)$ & $f\left(u_{3}\right)$ & Possible four edge labels \\
\hline \hline 1 & 2 & 3 & 4 & $1,2,1,1$ \\
\hline 1 & 2 & 4 & 3 & $1,3,1,2$ \\
\hline 1 & 3 & 2 & 4 & $2,1,1,1$ \\
\hline 1 & 3 & 4 & 2 & $2,3,1,3$ \\
\hline 1 & 4 & 2 & 3 & $3,1,1,2$ \\
\hline 1 & 4 & 3 & 2 & $3,2,1,3$ \\
\hline 2 & 1 & 3 & 4 & $1,1,2,1$ \\
\hline 2 & 1 & 4 & 3 & $1,2,2,2$ \\
\hline 2 & 3 & 1 & 4 & $1,1,2,1$ \\
\hline 2 & 3 & 4 & 1 & $1,2,2,4$ \\
\hline 2 & 4 & 1 & 3 & $2,1,2,2$ \\
\hline 2 & 4 & 3 & 1 & $2,1,2,4$ \\
\hline 3 & 1 & 2 & 4 & $2,1,3,1$ \\
\hline 3 & 1 & 4 & 2 & $2,1,3,3$ \\
\hline 3 & 2 & 1 & 4 & $1,2,3,1$ \\
\hline 3 & 2 & 4 & 1 & $1,1,3,4$ \\
\hline 3 & 4 & 1 & 2 & $1,2,3,3$ \\
\hline 3 & 4 & 2 & 1 & $1,1,3,4$ \\
\hline 4 & 1 & 2 & 3 & $3,2,4,2$ \\
\hline 4 & 1 & 3 & 2 & $3,1,4,3$ \\
\hline 4 & 2 & 1 & 3 & $2,3,4,2$ \\
\hline 4 & 2 & 3 & 1 & $2,1,4,4$ \\
\hline 4 & 3 & 1 & 2 & $1,3,4,3$ \\
\hline 4 & 3 & 2 & 1 & $1,2,4,4$ \\
\hline \hline & & & & \\
\hline \hline
\end{tabular}

Table-2: If $f\left(v_{2}\right)=5$

\section{References}

[1] A. Rosa, On certain valuation of graph, Theory of Graphs (Rome, July 1966), Goden and Breach, N. Y. and Paris, pp. 349-355, (1967).

[2] C. Hoede and H. Kuiper, All wheels are graceful, Util. Math., 14, pp. 311, (1987). 
[3] F. Harary, Graph theory, Narosa Publishing House, New Delhi, (2001).

[4] J. A. Gallian, The Electronics Journal of Combinatorics, 18, DS6, (2015).

\section{H. M. Makadia}

Lukhdhirji Engineering College,

Morbi-363642

e-mail: makadia.hardik@yahoo.com

\section{H. M. Karavadiya}

Labhubhai Trivedi Institute of Technology,

Rajkot - 360005

e-mail: hardik.karavadiya2016@gmail.com

and

\section{J. Kaneria}

Department of Mathematics, Saurashtra University,

Rajkot - 360005

e-mail: kaneriavinodray@gmail.com 